Institute for Evidence in Medicine, Medical Center - University of Freiburg, Faculty of Medicine University of Freiburg, Freiburg, Germany

2 Department of Physiology and Biochemistry of Nutrition, Max Rubner-Institut, Karlsruhe, Germany

3 Cochrane Germany, Cochrane Germany Foundation, Freiburg, Germany

Correspondence to: L Schwingshack schwingshackl@ifem.uni-freiburg.de Cite this as: BMJ 2020;370:m2417 http://dx.doi.org/10.1136/bmj.m2417 Published: 15 July 2020

\title{
The healthiness and sustainability of food based dietary guidelines
}

\author{
Are plant based diets better for human health and the planet? \\ Lukas Schwingshackl, ${ }^{1}$ Bernhard Watzl, ${ }^{2}$ Joerg J Meerpohl1, 3
}

Non-communicable diseases contributed to more than $70 \%$ of total deaths worldwide in $2017,{ }^{1}$ with suboptimal diet accounting for $15 \%$ of disability adjusted life years. ${ }^{2}$ Food based dietary guidelines inform consumers on adequate nutrition and health promotion and aim to reduce the burden of non-communicable diseases in a population by modifying food intake. ${ }^{34}$ They also guide optimal nutrient and energy supply in the context of dietary and cultural differences between countries. ${ }^{4}$

High adherence to recommended diets such as the Healthy Eating Index is usually associated with lower risk of non-communicable diseases and early death. ${ }^{5}$ But analysis of 34 European such guidelines found nothing on sustainability, ${ }^{4}$ and it is only recently that updates have started to also include environmental aspects of food production and consumption. ${ }^{6}$ Now, in a linked study, Springmann and colleagues (doi:10.1136/bmj.m2322) have modelled both the health and the environmental impacts of adopting global and national food based dietary guidelines ${ }^{7}$ and compared these with targets for global health and environmental outcomes, such as the non-communicable diseases agenda ${ }^{8}$ and the Paris Agreement on Climate Change. ${ }^{9}$

The authors collated and scored quantitative recommendations, such as "eat five servings of fruits and vegetables a day," from 85 national guidelines along with global guidelines from the World Health Organization $^{10}{ }^{11}$ and the EAT-Lancet Commission. ${ }^{12}$ They then used modelling to estimate how these recommendations could reduce premature mortality from chronic diseases. To estimate the impacts on health, 11 risk factors were used in the model, such as high consumption of red and processed meats, low consumption of plant based foods and fish, and body mass index. Disease endpoints included coronary heart disease, stroke, type 2 diabetes, cancer, and respiratory disease. To estimate the environmental implications of adopting national dietary guidelines, the authors considered country and crop specific footprints for greenhouse gas emissions, land and fresh water use, and application of nitrogen and phosphorus fertilisers.

The study's main dietary message is that public health strategies for nutrition should focus on increasing intake of whole grains, the food group that has previously been ranked highest in terms of reducing premature mortality, followed by fruits and vegetables. $^{213}$ Furthermore, the results confirm that most health benefits from adopting existing national guidelines would come from balancing energy intake and bodyweight. Lastly, the modelling showed that food related greenhouse gas emissions could be reduced by an average of $13 \%$ (550 million tonnes of carbon dioxide equivalent) across all countries, driven mostly by eating less beef and lamb: however, this would be offset by greater intake of milk and other dairy products. Overall, the authors estimate that adopting national food based dietary guidelines would lead to moderate reductions in premature mortality from non-communicable diseases (15\%) and mixed changes in demand for environmental resources. Adopting the EAT-Lancet recommendations could lead to a $25 \%$ reduction and much larger decreases in greenhouse gas emissions.

The study has several strengths but its limitations mean its findings should be interpreted with caution. Firstly, the modelling relied on the assumption that exposure-outcome relations are causal, yet the estimated measures of association could reflect residual confounding as they were based on data from meta-analyses of prospective cohort studies rather than from randomised controlled trials (which are rare in nutritional research). ${ }^{14}$ Secondly, although the study graded the certainty of evidence for the selected associations between risk and disease, using the comprehensive GRADE (grading of recommendations assessment, development and evaluation) approach could have improved both transparency and trustworthiness. ${ }^{15}$ Thirdly, the health impact of a food group is not determined solely by its associations with disease but by the provision of essential nutrients: for example, dairy products provide half of the daily calcium and iodine intake for many populations. ${ }^{16}$

Perhaps the most important finding from this study is the uncertainty that it highlights, not least about plant based foods. In overall terms the EAT-Lancet Commission proposals seem superior in terms of reducing mortality from non-communicable diseases and cutting greenhouse gas emissions. However, adopting the EAT-Lancet recommendations globally would not be affordable for many in low income countries without concomitant economic growth, improved local food production and supply, and expansion of the range of lower cost animal products, fruits, and vegetables. ${ }^{17}$ We still have some way to go before diets can become healthier and more sustainable worldwide.

Competing interests: The BMJ has judged that there are no disqualifying financial ties to commercial companies.

Provenance and peer review: Commissioned; not externally peer reviewed.

Roth GA, Abate D, Abate KH, etalGBD 2017 Causes of Death Collaborators. Global, regional, and national age-sex-specific mortality for 282 causes of death in 195 countries and territories, 1980-2017: a systematic analysis for the Global Burden of Disease Study 2017. Lancet 2018;392:1736-88. doi: 10.1016/S0140-6736(18)32203-7. pmid: 30496103 
2 GBD 2017 Diet Collaborators. Health effects of dietary risks in 195 countries, 1990-2017: a systematic analysis for the Global Burden of Disease Study 2017. Lancet 2019;393:1958-72. doi: 10.1016/S0140-6736(19)30041-8. pmid: 30954305

3 Schwingshackl L, Schlesinger S, Devleesschauwer B, etal. Generating the evidence for risk reduction: a contribution to the future of food-based dietary guidelines. Proc Nutr Soc 2018;77:432-44. doi: 10.1017/S0029665118000125. pmid: 29708078

4 Bechthold A, Boeing H, Tetens I, Schwingshackl L, Nöthlings U. Perspective: Food-Based Dietary Guidelines in Europe-Scientific Concepts, Current Status, and Perspectives. Adv Nutr 2018;9:544-60. doi: 10.1093/advances/nmy033. pmid: 30107475

5 Schwingshackl L, Bogensberger B, Hoffmann G. Diet Quality as Assessed by the Healthy Eating Index, Alternate Healthy Eating Index, Dietary Approaches to Stop Hypertension Score, and Health Outcomes: An Updated Systematic Review and Meta-Analysis of Cohort Studies. J Acad Nutr Diet 2018;118:74-100.e11. doi: 10.1016/j.jand.2017.08.024. pmid: 29111090

6 Brink E, van Rossum C, Postma-Smeets A, etal. Development of healthy and sustainable food-based dietary guidelines for the Netherlands. Public Health Nutr 2019;22:2419-35. doi: 10.1017/S1368980019001435. pmid: 31262374

7 Springmann M, Spajic L, Clark MA, etal. The healthiness and sustainability of national and global food based dietary guidelines: modelling study. BMJ 2020;369:m2322.

8 World Health Organization. Global NCD Action Plan 2013-2020. WHO, 2013.

9 United Nations Framework Convention on Climate Change. Report of the Conference of the Parties on its twenty-first session, held in Paris from 30 November to 13 December 2015 Addendum. Part two: Action taken by the Conference of the Parties at its twenty-first session. UNFCCC, 2016.

10 World Health Organization. Diet, nutrition and the prevention of chronic diseases: Report of the joint WHO/FAO expert consultation. WHO, 2003

11 World Health Organization. Human energy requirements: Report of a Joint FAO/WHO/UNU Expert Consultation, Rome, Italy, 17-24 October 2001. WHO, 2004.

12 Willett W, Rockström J, Loken B, etal. Food in the Anthropocene: the EAT-Lancet Commission on healthy diets from sustainable food systems. Lancet 2019;393:447-92. doi: 10.1016/S0140-6736(18)31788-4. pmid: 30660336

13 Schwingshackl L, Knüppel S, Michels N, etal. Intake of 12 food groups and disability-adjusted life years from coronary heart disease, stroke, type 2 diabetes, and colorectal cancer in 16 European countries. Eur J Epidemio/2019;34:765-75. doi: 10.1007/s10654-019-00523-4. pmid: 31030306

14 Pan A, Lin X, Hemler E, Hu FB. Diet and Cardiovascular Disease: Advances and Challenges in Population-Based Studies. Cell Metab 2018;27:489-96. doi: 10.1016/j.cmet.2018.02.017. pmid: 29514062

15 Guyatt G, Oxman AD, Akl EA, etal. GRADE guidelines: 1. Introduction-GRADE evidence profiles and summary of findings tables. / Clin Epidemiol 2011;64:383-94. doi: 10.1016/j.jclinepi.2010.04.026. pmid: 21195583

16 Haug A, Høstmark AT, Harstad OM. Bovine milk in human nutrition--a review. Lipids Health Dis 2007;6:25. doi: 10.1186/1476-511X-6-25. pmid: 17894873

17 Hirvonen K, Bai Y, Headey D, Masters WA. Affordability of the EAT-Lancet reference diet: a global analysis. Lancet Glob Health 2020;8:e59-66. doi: 10.1016/S2214-109X(19)30447-4. pmid: 31708415 